\title{
Red ruby glass from gold nanoparticles obtained by LASiS - a new approach.
}

J.C. Almeida ${ }^{1 *}$, E.J.C. Davim³ ${ }^{3}$ I.M. Miranda Salvado ${ }^{1}$, M.H.V. Fernandes ${ }^{1}$, F.M.

Costa $^{2}$, F. Rey-García ${ }^{2}$, L.M. Francisco ${ }^{3}$, A.B. Lopes ${ }^{1}$

${ }^{1}$ Department of Materials and Ceramic Engineering, CICECO, Universidade de Aveiro, 3810-193Aveiro, Portugal, ${ }^{2} / 3 \mathrm{~N}$, Department of Physics, Universidade de Aveiro, 3810-193 Aveiro, Portugal, ${ }^{3}$ Vista Alegre Atlantis S.A., Zona Industrial do Casal da Areia, Casal da Areia, 2460-392 Alcobaça, Portugal

* Corresponding author at: Tel.: +351 234370354; fax: +351 234370204; E-mail address: jcalmeida@ua.pt

Keywords: laser, ablation, LASiS, sol-gel, red glass

\begin{abstract}
Currently, most of the red colored glasses are still produced using cadmium sulfoselenide, despite its high toxicity. Alternative solutions, more environmentally friendly, have been sought, most of them going through the use of gold or copper nanoparticle, the color being obtained by striking.

This work presents a new methodology for production of a red colored glass by the incorporation of gold nanoparticles in the melt, trying to take advantage of an amorphous silica layer involving the nanoparticles. Gold nanoparticles (AuNP) were produced by laser ablation synthesis in solution (LASiS), using a solution of tetraethyl orthosilicate (TEOS) and isopropanol (IPA).
\end{abstract}

A xerogel was prepared using the TEOS:IPA:AuNp suspension, mixed with a alkaline-earth aluminosilicate colorless glass and melted at $1350 \stackrel{\circ}{\circ} \mathrm{C}$ producing a glass with an intense and homogeneous ruby red color without the need of a striking stage.

\section{Introduction}

Most of the ruby red glasses are still produced using cadmium selenide in its composition. Due to cadmium toxicity, hardly tolerated in the context of environmental protection, problems related with its migration become a relevant issue particularly in food and beverage container industry. In use since 1984, the European Directive 84/500/EEC sets the limits for lead and cadmium migration from ceramics depending 
on the article category. For larger container categories, for example, migration range currently varies from 1.5 to $4 \mathrm{mg} / \mathrm{L}$ for lead and 0.1 to $0.3 \mathrm{mg} / \mathrm{L}$ for cadmium. However, this reality will change soon. In fact, the new regulation, "Food safety - heavy metals in ceramic, glass and enameled table and kitchenware", has already been reviewed by representatives of the European ceramic and glass industry, and will be approved by the end of the first quarter of 2020. Cadmium selenide-based compounds are expected to be completely banned or their use very restricted.

Not being new [1], alternative solutions, more environmentally friendly, have been pursued and tested, most of them going through the use of gold or copper nanoparticles.

In both ruby glasses, whether obtained from cadmium selenide or from gold, the coloring is due to the existence of very small colloidal particles with sizes around 20 to $50 \mathrm{~nm}$. When there is an incident light, one part of the spectrum is absorbed by the colloidal particles while the rest of the spectrum is scattered according to Mie's theory [2]. In the case of ruby glasses, whether for gold, copper or even cadmium selenide, the absorption spectra are very similar, with the absorption band between 530 and 560 $\mathrm{nm}[3]$.

Known from antiquity, taking the case of the famous Lycurgus Cup manufactured between the $5^{\text {th }}$ and $4^{\text {th }}$ century B.C., ruby glass obtained with gold nanoparticles is produced introducing in the batch gold in its ionic form (usually using chloroauric acid, $\mathrm{HAuCl}_{4}$ ). During the glass melting, gold will be dissolved as ionic $\mathrm{Au}^{+}$, and due to its high electrochemical potential it will be easily reduced to its metallic form $\mathrm{Au}^{0}$. Since the ruby color depends on the size of the colloidal particles, it is necessary, in addition to a reducing medium, to ensure a good diffusion of the ions, which is often only succeeded with subsequent heat treatment (striking).

In order to reduce the effect of fusion atmosphere on the final color or reduce or even eliminate the need for a striking step, the introduction of gold nanoparticles along with the other glass components arose as a possible improvement.

Due to their increasing applicability, from catalysts to biomedicine, several gold nanoparticle production methodologies have emerged in recent years, many of which are extremely complex and costly, usually based on reduction reactions [4].

Among the various methodologies the simplest by far is laser ablation, considered a green technique due to the possibility of producing metallic nanoparticles in an aqueous medium; a metal plate target is ablated by a high-power laser and nanoparticles are formed in a medium (usually a liquid) that surrounds the target. Given that a liquid medium is present this technique is also called laser ablation synthesis in solution (LASiS). The most important parameters to control are energy, 
wavelength and pulse characteristics of the laser, ablation time, and the absorption of the liquid medium [5]. Adjusting the medium used it is also possible to control the size distribution of the produced nanoparticles and the stability of the suspension [6-8].

When comparing LASiS with chemical reduction methods (CRM) for large scale production, it should be noticed that LASiS has some limitations: (i) controlling the average size and size distribution of nanoparticles is difficult; (ii) the nanoparticles production rate, which is about $1-5 \mathrm{mg} \mathrm{h}^{-1}$ with a nanosecond laser, can be too low if mass production in short times is required; (iii) the start-up costs due to the laser and laser equipment can be excessive when a chemical laboratory is already present. As advantages, LASiS requires: (i) minimum manual operation; (ii) simple raw materials (metal plates) for the synthesis; (iii) minimum waste production during the synthesis procedure [6]. In addition, one of the most attractive benefits of using LASiS in the production of metal nanoparticles is the possibility of functionalizing the material during the ablation process [9-14].

The main goal of this work is to obtain a homogeneous red ruby glass using gold nanoparticles (AuNP) protected by an amorphous silica layer that competes against their dissolution during the melting and avoids the need of the striking stage. Such gold nanoparticles (AuNP) were produced by laser ablation synthesis in solution, using a solution of tetraethyl orthosilicate (TEOS) and isopropanol (IPA) and mixed as a xerogel with a powdered colorless glass.

\section{Experimental Procedure}

\subsection{Gold nanoparticles production by LASiS}

Tetraethyl orthosilicate (TEOS) and isopropanol (IPA from Sigma-Aldrich were used to prepare $50 \mathrm{ml}$ of a solution containing $1: 10 \mathrm{~mol} / \mathrm{mol}$ of TEOS:IPA, which is used as the liquid medium for the LASiS. A metallic gold plate was used as target. The ablation was performed using a Nd:YAG laser $(1064 \mathrm{~nm})$ system with a pulse duration and frequency of $10 \mathrm{~ns}$ and $10 \mathrm{~Hz}$, respectively (Figure 1). The pulsed laser was applied for 3 minutes, time enough to produce a concentrated suspension of gold nanoparticles (Figure 2) without the ignition of the isopropanol. 


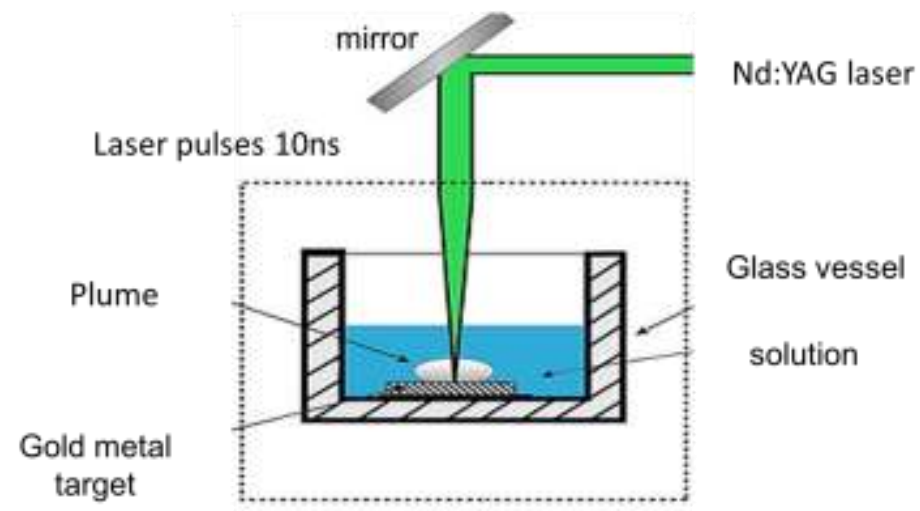

Figure 1: Laser ablation apparatus.

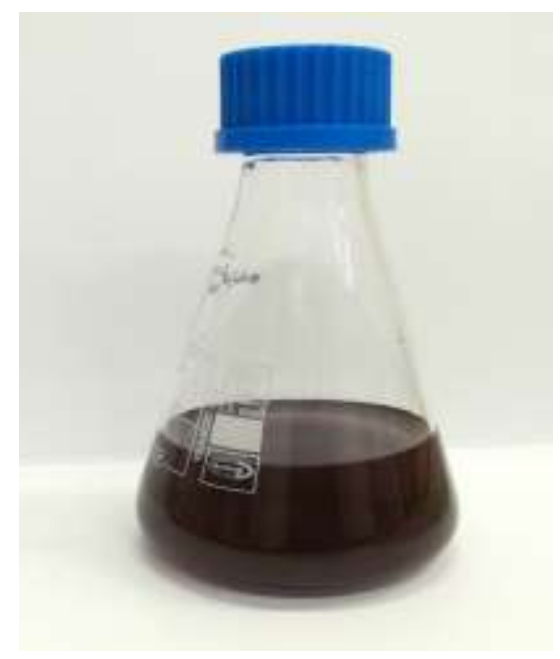

Figure 2: Gold nanoparticles suspension.

\subsection{Mixed glass $+\mathrm{SiO}_{2}+\mathrm{AuNP}$ sol-gel preparation}

In order to guarantee the hydrolysis of TEOS [15], $0.36 \mathrm{ml}$ of distilled water (1:4 $\mathrm{mol} / \mathrm{mol}$ of TEOS: $\mathrm{H}_{2} \mathrm{O}$ ) was added to $5 \mathrm{ml}$ of AuNP suspension, and poured over $10 \mathrm{~g}$ of a powdered alkaline-earth aluminosilicate colorless glass, supplied by the glassware producer Vista Alegre Atlantis, previously ground (particle size below $1 \mathrm{~mm}$ ) in an agate mortar.

After 24 hours at $60^{\circ} \mathrm{C}$, all the isopropanol was evaporated and an amorphous silica xerogel was obtained. The mixture of the xerogel and glass was ground again until a fine powder was obtained (size smaller than $250 \mu \mathrm{m}$ ) in order to ensure a good mixing. 


\subsection{Ruby red glass preparation}

The mixture prepared in 2.2. was added to more colorless glass powder in a mass proportion of 1:4, ground again ( $1 \mathrm{~g}$ of mixture added to $4 \mathrm{~g}$ of glass), and placed in a platinum crucible. The final mixture was fired at two different temperatures, $1450{ }^{\circ} \mathrm{C}$ and $1350 \stackrel{\circ}{\circ}$ for 1 hour and the melts quenched into a brass mould. It was observed that at $1450 \stackrel{\circ}{ } \mathrm{C}$ the glass was colorless with no signs of the presence of nanoparticles, indicating that they had dissolved in the melt. However, the mixture cast at $1350{ }^{\circ} \mathrm{C}$ was ruby red without the need of any striking treatment. In order to have a comparison basis, equivalent compositions were prepared using a solution of gold (III) chloride trihydrate and cadmium sulfoselenide $(\mathrm{Cd}(\mathrm{S}, \mathrm{Se}))$, as supplied by Vista Alegre Atlantis.

\subsection{Characterization}

The particle size of the AuNP in the TEOS:IPA suspension was evaluated using a Scanning Transmission Electron Microscope (STEM), Hitachi SU-70, in transmission mode, and image processing software (ImageJ). A 100 times diluted solution was prepared using isopropanol, and a $3.05 \mathrm{~mm}$ diameter copper TEM grid (300 mesh), Formvar/carbon coated, used for the observation. Three different images with 200k of magnification were used to the determination of a medium diameter. Gold concentration in the solution was determined by Inductively Coupled Plasma (ICP) spectroscopy using a ICP-OES Jobin Yvon Activa M spectrometer with a resolution of $4 \mathrm{ppb}$ for Au. UV-visible absorption spectra were obtained between 190 to $2700 \mathrm{~nm}$, using a step of $12 \mathrm{~nm}$ and a resolution of $0.1 \mathrm{~nm}$, in a Shimadzu UV-3100 spectrometer with $2 \mathrm{~mm}$ optical path quartz cells.

Color was determined in the quenched glasses prepared with the AuNP-LASiS particles, gold (III) chloride trihydrate and with cadmium sulfoselenide ( $\mathrm{Cd}(\mathrm{S}, \mathrm{Se}))$, using the CIE L*a* $\mathrm{b}^{*}$ chromatic coordinates on a Konica Minolta Chroma Meter CR-400, using D65 illuminant and 10ำ standard observer (Y:94.0, x:0.3156, y:0.3319).

\section{Results and Discussion}

Scanning transmission electron microscopy (STEM) observation of suspension samples prepared in the LASiS apparatus was carried out in transmission mode (Figure 3). The image analysis performed using the ImageJ software reveals that the average size of the AuNP is around $20( \pm 5) \mathrm{nm}$. The gold concentration in the solution, determined by Inductively Coupled Plasma (ICP), was 17.6 ppm. 


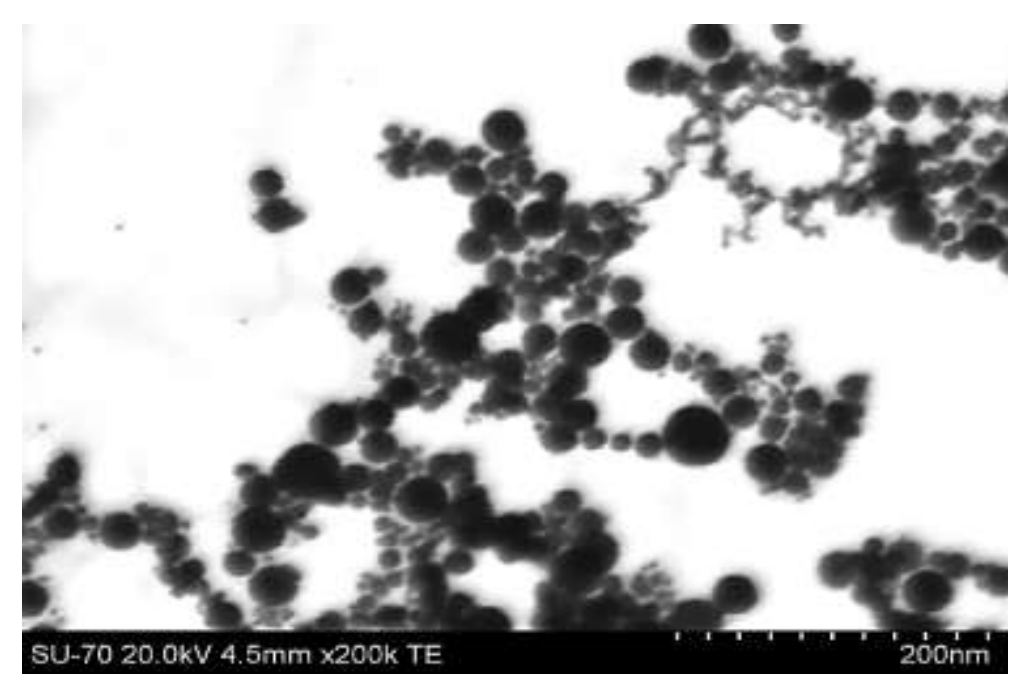

Figure 3: AuNP particles obtained by LASiS observed in STEM transmission mode.

UV-visible absorption spectra of the TEOS:IPA solution with the AuNP (Figure 4) reveals a broad peak ca. $510 \mathrm{~nm}$, a value near to the one $(520 \mathrm{~nm})$ reported as responsible to the deep-red color of AuNP with a size near $20 \mathrm{~nm}$ sols in water and glasses and due to the surface plasmon band (SPB).The SPB results from the collective oscillations of the electrons at the surface of the nanoparticles that is correlated with the electromagnetic field of the incoming light, i.e., the excitation of the coherent oscillation of the conduction band [16].

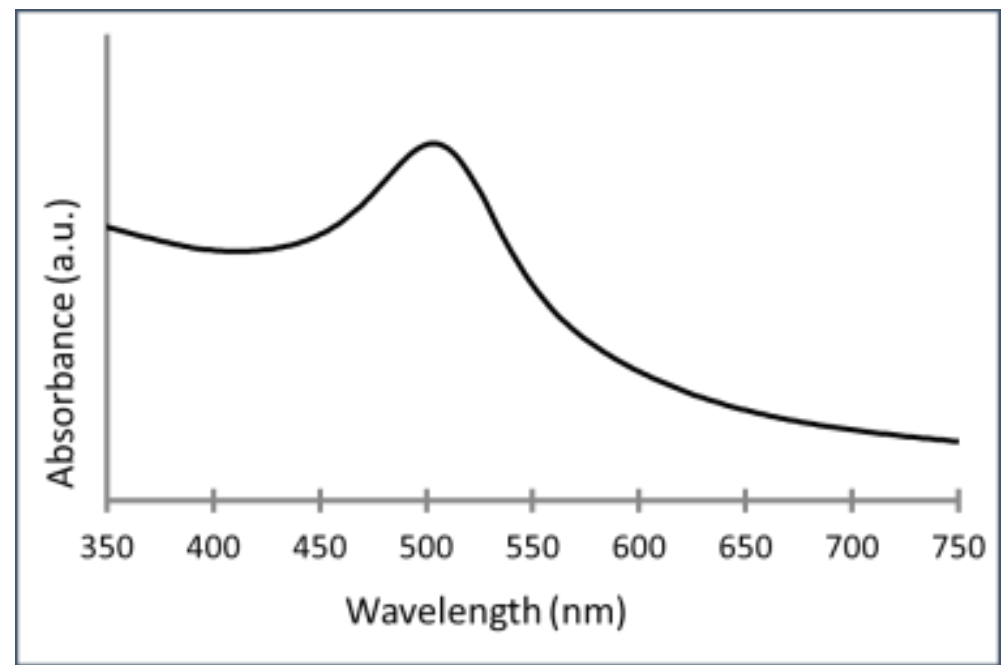

Figure 4: UV-visible spectrum of the TEOS:IPA:AuNP solution. 
According to Daniel and Astruc [4] AuNPs of mean diameter of 9, 15, 22, 48, and 99 $\mathrm{nm}$, exhibit SPB maximum $\lambda_{\max }$ at $517,520,521,533$, and $575 \mathrm{~nm}$, respectively, in aqueous media, with the SPB maximum and bandwidth influenced by the particle shape, medium refractive index, dielectric constant, and temperature.

Color was determined in the quenched glasses prepared with the AuNP-LASiS particles, gold (III) chloride trihydrate and with cadmium sulfoselenide $(\mathrm{Cd}(\mathrm{S}, \mathrm{Se}))$ using the CIE L* $a^{*} b^{*}$ chromatic coordinates. The results are depicted in Table 1.

Table 1: CIE L*a* $\mathrm{b}^{*}$ chromatic coordinates and quenched $\left(1350^{\circ} \mathrm{C}\right)$ glass photos.

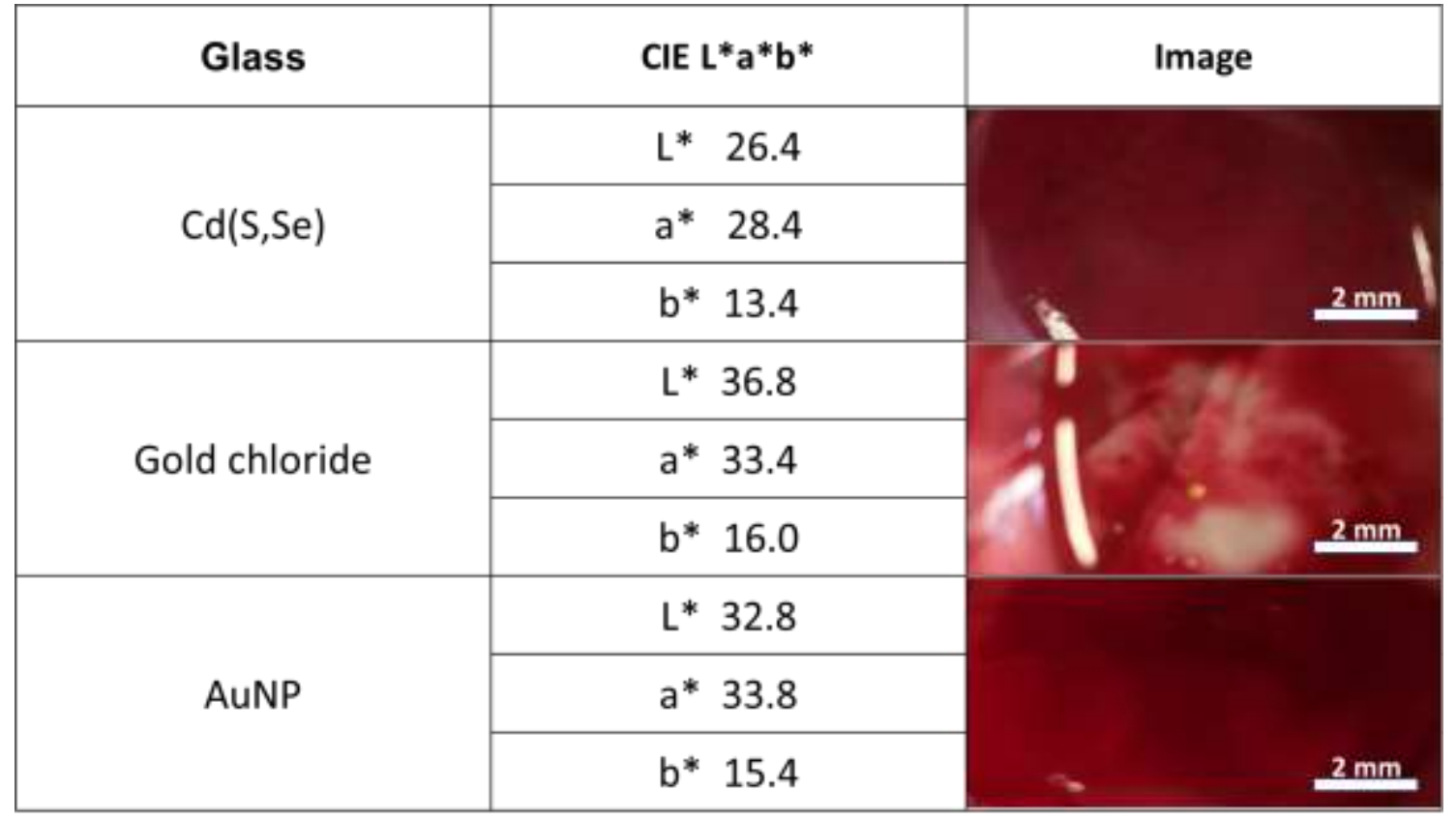

Image in Table 1 clearly shows that the color of the ruby red glass obtained from AuNP without a striking process is very similar to the one produced by Vista Alegre Atlantis with the addition of $\mathrm{Cd}(\mathrm{S}, \mathrm{Se})$. Glasses prepared with gold chloride showed a pink color with heterogeneous intensity.

This finding is very important once it proves that it is possible to have a good distribution of AuNP obtained by LASiS in the glass melted at $1350{ }^{\circ} \mathrm{C}$ without a deterioration of the nanoparticles, as a result of the presence of an amorphous silica phase embedding them. Besides a better color homogeneity is obtained when compared to the one obtained by addition of gold chloride. This opens up a wide range of possibilities in industrial practice, including the addition of a AuNP-containing mixture to the colorless glass melt in the kiln feeders, where the temperature is usually around $1350 \stackrel{\circ}{\circ}$. 


\section{Conclusions}

Laser ablation of $\mathrm{Au}$ in TEOS:IPA solution allowed to obtain AuNP in the suspension with an average size of around $20 \mathrm{~nm}$. This suspension was used to prepare by sol-gel an amorphous silica media involving the AuNP. The obtained xerogel was mixed with a colorless glass and melted at $1350 \stackrel{\circ}{\circ} \mathrm{C}$ producing a glass with an intense and homogeneous ruby red colour without the need of a striking stage. The observed improvement opens up the possibility of replicating the process in industrial practice, in particular the addition of AuNP-containing mixture to the colorless glass melt in the kiln feeders, where the temperature is usually around $1350^{\circ} \mathrm{C}$.

\section{Acknowledgments}

This work was developed within the scope of the project DecorGlass - Decoration Techniques for the Glass, PT2020 - SI I\&DT - I\&D copromotion no 3485, and by the project CICECO-Aveiro Institute of Materials, POCI-01-0145-FEDER-007679 (FCT Ref. UID/CTM/50011/2013), financed by national funds through the FCT/MEC and when appropriate cofinanced by FEDER under the PT2020 Partnership Agreement.

\section{References}

[1] J.M. Fernández Navarro, El vidrio, Consejo Superior de Investigaciones Científicas : Sociedad Espanola de Ceramica y Vidrio, 2003.

[2] G. Mie, Beiträge zur Optik trüber Medien, speziell kolloidaler Metallösungen, Ann. Phys. 330 (1908) 377-445. doi:10.1002/andp.19083300302.

[3] R.J. Santos Barros, Estabilização da cor vermelha no vidro industrial, University of Aveiro, 2014. https://ria.ua.pt/bitstream/10773/13653/1/Estabilizacao da cor vermelha no vidro industrial.pdf (accessed October 17, 2019).

[4] M.-C. Daniel, D. Astruc, Gold Nanoparticles: Assembly, Supramolecular Chemistry, Quantum-Size-Related Properties, and Applications toward Biology, Catalysis, and Nanotechnology, Chem. Rev. 104 (2004) 293-346. doi:10.1021/CR030698+.

[5] A. Reza Sadrolhosseini, M. Adzir Mahdi, F. Alizadeh, S. Abdul Rashid, Laser Ablation Technique for Synthesis of Metal Nanoparticle in Liquid, in: Laser Technol. Its Appl., IntechOpen, 2019: pp. 63-83. doi:10.5772/intechopen.80374.

[6] V. Amendola, M. Meneghetti, Laser ablation synthesis in solution and size manipulation of noble metal nanoparticles, Phys. Chem. Chem. Phys. 11 (2009) 3805. doi:10.1039/b900654k.

[7] V. Amendola, M. Meneghetti, What controls the composition and the structure of nanomaterials generated by laser ablation in liquid solution?, Phys. Chem. Chem. Phys. 15 (2013) 3027-3046. doi:10.1039/C2CP42895D. 
[8] S. Scaramuzza, S. Agnoli, V. Amendola, Metastable alloy nanoparticles, metal-oxide nanocrescents and nanoshells generated by laser ablation in liquid solution: influence of the chemical environment on structure and composition, Phys. Chem. Chem. Phys. 17 (2015) 28076-28087. doi:10.1039/C5CP00279F.

[9] T. Salminen, M. Honkanen, T. Niemi, Coating of gold nanoparticles made by pulsed laser ablation in liquids with silica shells by simultaneous chemical synthesis, Phys. Chem. Chem. Phys. 15 (2013) 3047-3051. doi:10.1039/C2CP42999C.

[10] A. Ancona, M.C. Sportelli, A. Trapani, R.A. Picca, C. Palazzo, E. Bonerba, F.P. Mezzapesa, G. Tantillo, G. Trapani, N. Cioffi, Synthesis and characterization of hybrid copper-chitosan nano-antimicrobials by femtosecond laser-ablation in liquids, Mater. Lett. 136 (2014) 397-400. doi:10.1016/J.MATLET.2014.08.083.

[11] Z. Sheykhifard, M. Ranjbar, H. Farrokhpour, H. Salamati, Direct Fabrication of $\mathrm{Au} / \mathrm{Pd}$ (II) Colloidal Core-Shell Nanoparticles by Pulsed Laser Ablation of Gold in $\mathrm{PdCl} 2$ Solution, J. Phys. Chem. C. 119 (2015) 9534-9542. doi:10.1021/jp512582q.

[12] D.M. Arboleda, J.M.J. Santillán, V.B. Arce, M.B. Fernández van Raap, D. Muraca, M.A. Fernández, R.M. Torres Sanchez, D.C. Schinca, L.B. Scaffardi, A simple and "green" technique to synthesize long-term stability colloidal Ag nanoparticles: Fs laser ablation in a biocompatible aqueous medium, Mater. Charact. 140 (2018) 320-332. doi:10.1016/J.MATCHAR.2018.04.021.

[13] M. Sportelli, M. Izzi, A. Volpe, M. Clemente, R. Picca, A. Ancona, P. Lugarà, G. Palazzo, N. Cioffi, The Pros and Cons of the Use of Laser Ablation Synthesis for the Production of Silver Nano-Antimicrobials, Antibiotics. 7 (2018) 67. doi:10.3390/antibiotics7030067.

[14] K. Tibbetts, Laser ablation in liquid: A powerful route to new nanoparticle catalysts, Res. Outreach. (2019) 50-53. doi:10.32907/ro-106-5053.

[15] C.J. Brinker, G.W. Scherer, Sol-gel science : the physics and chemistry of sol-gel processing, Academic Press, Boston, 1990. http://www.loc.gov/catdir/description/els032/89015631.html.

[16] M.A. Garcia, Surface plasmons in metallic nanoparticles: fundamentals and applications, J. Phys. D. Appl. Phys. 44 (2011) 283001. doi:10.1088/0022-3727/44/28/283001. 